\title{
Vinylbenzyl-Type Polyamide Macromer Synthesized from Bicyclic Oxalactam. Preparation and Characterization of Graft Copolymer Composed of Polystyrene as a Stock and Hydrophilic Polyamide as Branches
}

\author{
Kazuhiko Hashimoto, Hiroshi Sumitomo, and Masaya KAWASUmi \\ Faculty of Agriculture, Nagoya University, Chikusa-ku, Nagoya 464, Japan
}

(Received December 20, 1984)

\begin{abstract}
Vinylbenzyl-type polyamide macromers (1) of various average molecular weights $\left(M_{n} ; 3900,5400\right.$, and 8400$)$ were prepared by the anionic ring-opening polymerization of a bicyclic oxalactam, 8-oxa-6-azabicyclo[3.2.1] octan-7-one (abbreviated as BOL, 2) followed by a reaction with $p$-vinylbenzylamine. The relative reactivity of the macromer $\left(\mathbf{M}_{1}\right)$ in the radical copolymerization with styrene $\left(\mathrm{M}_{2}\right)$ was found to be lower than that of $p$-methylstyrene from the estimation of the reciprocal monomer reactivity ratio $\left(1 / r_{2}\right)$. The resulting graft copolymer composed of a polystyrene stock and polyamide branches was characterized by structural analysis, solubility test, and determination of moisture sorption. Two kinds of transparent binary blend films, which were i) the graft copolymer and homopolystyrene and ii) the graft copolymer and polyBOL, were cast on a glass plate from the corresponding chloroform and chloroform-methanol $(9: 1)$ solutions, respectively. In the case of the former film comprising mainly polystyrene, the hydrophilic polyamide segment of the graft copolymer as a really minor component was found to be located on the glassside surface from the contact angle measurement and the attenuated total reflection-infrared spectroscopy.

KEY WORDS Bicyclic Oxalactam / 8-Oxa-6-azabicyclo[3.2.1]octan-7-one /

Polyamide / Macromer / Graft Copolymer / Blend / Film / Surface /
\end{abstract}

Bicyclic oxalactam, 8-oxa-6-azabicyclo[3.2.1] octan-7-one (abbreviated as BOL, 2) can be easily polymerized using anionic catalysts under mild conditions such as room temperature in dimethyl sulfoxide to give a high molecular weight polyamide $3 .^{1-3}$ The resulting polyBOL is cast to the hygroscopic membrane which has an excellent permeability and permselectivity for water and solutes of different sizes in an aqueous solution, respectively. ${ }^{2,4-6}$ Such a novel polyamide is expected to be available to prepare block- and graft copolymers. We have synthesized not only a block copolymer having the polyamide and polyoxyethylene segments ${ }^{7}$ but also polyamide macromers having telechelic amino groups and a vinylbenzyl group, respectively. ${ }^{8-10}$ In the present paper, the vinyl-

benzyl-type polyamide macromers of various molecular weights were synthesized from BOL. A novel graft copolymer having a polystyrene stock and hydrophilic polyamide branches was also prepared by the radical copolymerization with styrene.

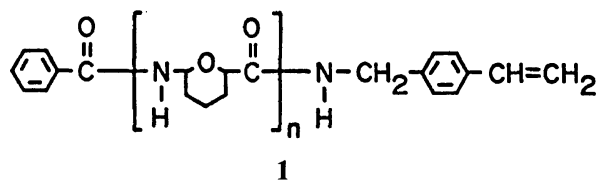

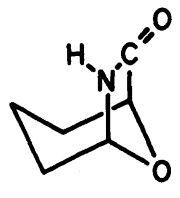

2

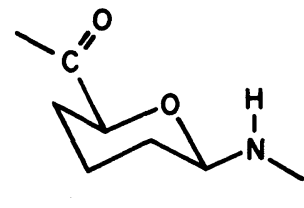

3 


\section{EXPERIMENTAL}

\section{Reagents}

BOL (2) was prepared by the same procedure as reported earlier, ${ }^{1,2}$ and stored over phosphorus pentoxide in vacuo until use. $N$ Benzoyl-8-oxa-6-azabicyclo[3.2.1]octan-7-one, potassium pyrrolidonate, and $p$-vinylbenzylamine (abbreviated as BzBOL, K-Pyrdn, and VBA, respectively) were prepared as described in the literature..$^{9,11,12}$ Dimethyl sulfoxide $\left(\mathrm{Me}_{2} \mathrm{SO}\right), N, N$-dimethylformamide (DMF), and pyridine were dried over calcium hydride, magnesium sulfate, and sodium hydroxide, respectively, and distilled under reduced or atmospheric pressure. Styrene and azobisisobutyronitrile (AIBN) were purified by the same method as described in the previous article. ${ }^{9} \mathrm{~N}$-(2-Tetrahydropyranyl)benzamide (4) was prepared from benzamide $(6.1 \mathrm{~g})$ and 3,4-dihydro-2 $\mathrm{H}$-pyran $(4.2 \mathrm{~g})$ as reported by Speziale et al., ${ }^{13}$ and recrystallized from the benzene solution: yield $3.6 \mathrm{~g}$ $\left(35^{\circ} \%\right) ; \mathrm{mp}, 122-125^{\circ} \mathrm{C}$ (lit. $\left.{ }^{13} 124-125^{\circ} \mathrm{C}\right) . \mathrm{N}$ ( $p$-Vinylbenzyl)acetamide (5) was obtained by the reaction of VBA $(0.47 \mathrm{~g})$ and acetic anhydride $(0.47 \mathrm{~g})$ in ethyl acetate $(10 \mathrm{ml})$ at room temperature for $1 \mathrm{~h}$ and recrystallized from the diethyl ether solution: yield, $0.46 \mathrm{~g}$ $(74 \%)$; mp, $103.5-104^{\circ} \mathrm{C}$. Elemental analysis. Calcd. for $\mathrm{C}_{11} \mathrm{H}_{13} \mathrm{NO}$ : C, $75.40 \%$; $\mathrm{H}, 7.48 \%$; $\mathrm{N}, 7.99 \%$. Found: C, $75.70 \%$;, $7.50 \%$; N, $7.91 \%$.

\section{Preparation of Vinylbenzyl-Type Polyamide Macromer (1) \\ Under dry nitrogen, $6.4 \mathrm{~g}$ of BOL and $0.06 \mathrm{~g}$} of K-Pyrdn were dissolved in $8 \mathrm{~g}$ of $\mathrm{Me}_{2} \mathrm{SO}$ in a flask. Immediately a BzBOL solution $(0.35 \mathrm{~g}$ in $12 \mathrm{~g}$ of $\mathrm{Me}_{2} \mathrm{SO}$ ) was poured into the solution with stirring. After the polymerization at $25^{\circ} \mathrm{C}$ for $30 \mathrm{~min}$, an excess of VBA solution in $\mathrm{Me}_{2} \mathrm{SO}(0.6 \mathrm{~g}$ VBA in $5 \mathrm{~g}$ of the solvent) was directly added to the polymerization system and the flask was allowed to stand in a bath controlled at $25^{\circ} \mathrm{C}$ for $6 \mathrm{~h}$. After quenching the reaction mixture by the addition of acetic acid $(0.5 \mathrm{ml})$, volatile components were evaporated under reduced pressure below $30^{\circ} \mathrm{C}$. The residue was poured into a large amount of acetone. The resulting colorless polymer was collected on a glass filter, washed with acetone, and dried in vacuo: yield $5.0 \mathrm{~g} ; M_{n} 5400$.

\section{Radical Homopolymerization of the Polyamide Macromer}

The polyamide macromer $\left(M_{n} 5400,0.50 \mathrm{~g}\right)$ and AIBN $(3.5 \mathrm{mg}$ ) were dissolved in $2.3 \mathrm{~g}$ of DMF in an ampoule. After degassing four times in a cooling bath, the ampoule was sealed and kept at $60^{\circ} \mathrm{C}$ for 2 days. The polymerization mixture was poured into a large amount of acetone. The gross polymer formed and the unreacted macromer were collected, washed with acetone, and dried in vacuo. The conversion was determined by gel permeation chromatography.

\section{Radical Copolymerization of the Polyamide Macromer with Styrene}

The polyamide macromer $\left(M_{n} 3900,0.50 \mathrm{~g}\right)$, styrene $(1.48 \mathrm{~g})$, and AIBN (4.8 mg) were dissolved in $1.5 \mathrm{~g}$ of DMF in an ampoule. The polymerization was carried out in the same manner as in the homopolymerization. After the polymerization at $60^{\circ} \mathrm{C}$ for 1 day, the polymerization mixture was poured into a large amount of acetone. The precipitate containing the graft copolymer and the unreacted macromer was collected, washed with acetone, and dried in vacuo. The conversion of styrene was calculated from the weight of the recovered whole polymer and its composition estimated by ${ }^{1} \mathrm{H}$ NMR spectroscopy. The graft copolymer was separated from the unreacted macromer by the repeated fractional precipitation using chloroform and methanol as a diluent and a precipitant (the volume ratio, $1: 4)$, respectively. The unreacted macromer was recovered from the filtrate after the condensation and reprecipitation in a large amount of acetone. The conversion of the 
macromer was calculated from the conversion of styrene estimated above and the composition of the isolated copolymer determined by ${ }^{1} \mathrm{H}$ NMR spectroscopy. The composition was confirmed also by the elemental analysis.

\section{Characterization}

Gel permeation chromatogram was recorded on a Hitachi Model 634A high performance liquid chromatograph (column, Shodex GPCA $80 \mathrm{M}$ or GPCA $803 \rightarrow 804,8 \phi \times$ $1000 \mathrm{~mm}$; solvent, chloroform). ${ }^{1} \mathrm{H}$ and ${ }^{13} \mathrm{C}$ NMR spectra were obtained with a JEOL JNM-FX-200 Fourier transform high resolution spectrometer. The UV spectrum was taken with a JASCO UVIDEC-1 spectrophotometer. The DSC thermogram of the polymer was taken with a Perkin-Elmer Model DSC-2 differential scanning calorimeter. In order to estimate the moisture sorption, the previouslydried polymer samples were kept under a certain relative humidity at $20^{\circ} \mathrm{C}$ and the amount of the adsorbed water was traced gravimetrically for more than a week until it reached a constant value. Relative humidity was controlled by varying the weight ratio of concentrated sulfuric acid to water. The two kinds of binary blend films were prepared by casting and drying up at room temperature from i) $3 \%$ chloroform solution of the graft copolymer and homopolystyrene and ii) 3\% chloroform-methanol $(9: 1)$ solution of the graft copolymer and polyBOL. The contact angle of a water droplet on the film was measured at $25^{\circ} \mathrm{C}$ with a Model CA-A telescopic goniometer made by Kyowa Scientific Industries Co., Tokyo. The attenuated total reflection-infrared spectrum of the blend film was recorded on a JASCO A-3 infrared spectrophotometer equipped with a ATR-6 attenuated all reflection apparatus using KRS-5 prisms.

\section{RESULTS AND DISCUSSION}

\section{Synthesis of the Vinylbenzyl-Type Polyamide Macromer}

For design of a graft copolymer having hydrophilic polyamide branches, the vinylbenzyl-type polyamide macromer was first prepared from BOL as described in the previous short communication. ${ }^{9}$ Thus, anionic polymerization of $\mathrm{BOL}$ using various amounts of $\mathrm{BzBOL}$ as an activator was carried out in $\mathrm{Me}_{2} \mathrm{SO}$ at $25^{\circ} \mathrm{C}$ for $30 \mathrm{~min}$ and an excess of VBA was directly added to the solution. The mixture was allowed to stand at $25^{\circ} \mathrm{C}$ for more than $5 \mathrm{hr}$ to let the generated acyllactam-type growing end react with VBA (eq 1).

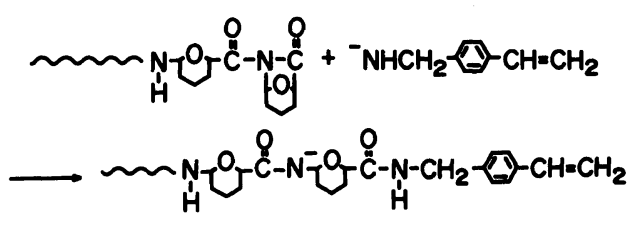

A typical ${ }^{1} \mathrm{H}$ NMR spectrum of the resulting polymer is shown in Figure 1, from which not only proton peaks due to the repeating $\mathrm{BOL}$ unit but also many peaks assignable to both terminal groups can be seen. The chemical shifts of these characteristic peaks were found to correspond exactly to those in the spectra of their model compounds, $N$-(2-tetrahydropyranyl)benzamide (4) and $N$-( $p$-vinylbenzyl)acetamide (5). Therefore all peaks can be as-

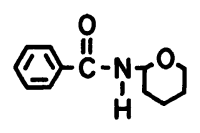

4

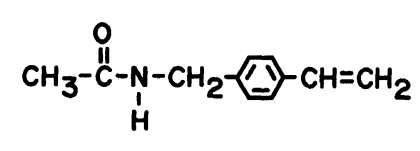

5

signed as indicated in the molecular formula in Figure 1. 


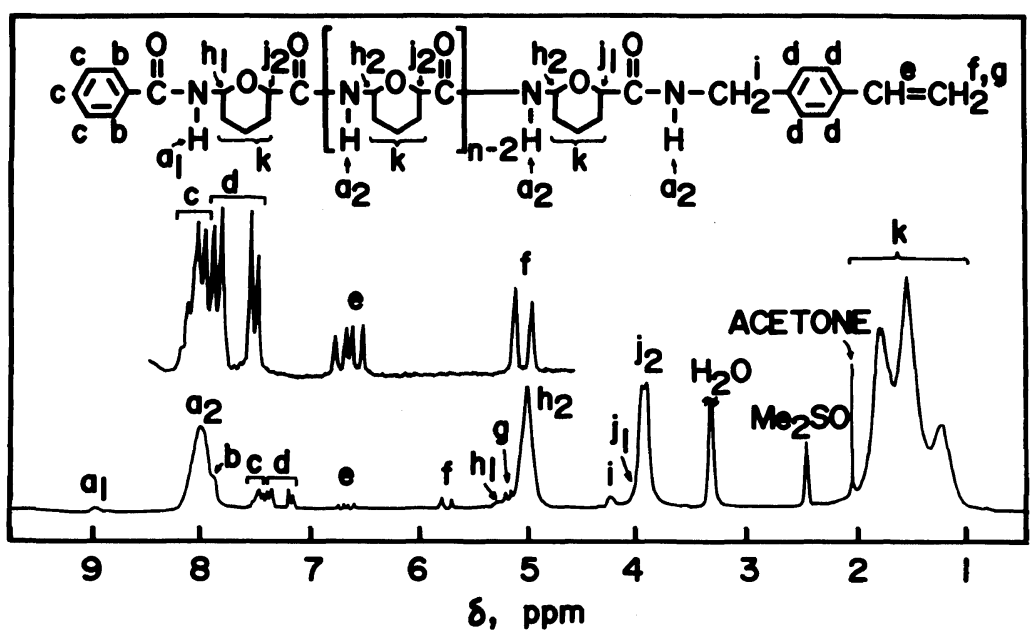

Figure 1. ${ }^{1} \mathrm{H}$ NMR spectrum of the polyamide macromer obtained by anionic polymerization of $\mathrm{BOL}$ followed by the reaction with $p$-vinylbenzylamine $\left(M_{n}, 3800 ; 9.5 \% \mathrm{Me}_{2} \mathrm{SO}-d_{6}\right.$ solution; TMS; room temperature, $200 \mathrm{MHz}$ ).

Table I. Synthesis of polyamide macromers by anionic polymerization of BOL followed by reaction with $p$-vinylbenzylamine ${ }^{a}$

\begin{tabular}{|c|c|c|c|c|c|c|c|}
\hline \multirow{2}{*}{$\frac{\mathrm{BzBOL}}{\mathrm{mol} \% \text { to } \mathrm{BOL}}$} & \multirow{2}{*}{\begin{tabular}{c}
\multicolumn{1}{c|}{$\mathrm{VBA}^{\mathrm{b}}$} \\
$\begin{array}{l}\text { Mole ratio } \\
\text { to } \mathrm{BzBOL}\end{array}$
\end{tabular}} & \multirow{2}{*}{$\begin{array}{c}\begin{array}{c}\text { Acetone insoluble } \\
\text { polymer }\end{array} \\
\mathrm{g}\end{array}$} & \multirow{2}{*}{$n^{\mathrm{c}}$} & \multicolumn{2}{|c|}{$x^{d}$} & \multirow{2}{*}{$M_{n}^{\mathrm{c}}$} & \multirow{2}{*}{$\frac{M_{w}^{8}}{M_{n}}$} \\
\hline & & & & ${ }^{1} \mathrm{H} \mathrm{NMR}^{\mathrm{e}}$ & $U V^{f}$ & & \\
\hline 5.0 & 2.1 & 3.9 & 29 & $0.9_{8}$ & $0.9_{8}$ & 3900 & $1.1_{9}$ \\
\hline 3.0 & 3.0 & 5.0 & 40 & $1.0_{1}$ & $1.0_{9}^{\circ}$ & 5400 & 1.29 \\
\hline 1.5 & 3.9 & 5.5 & 64 & $1.0_{3}$ & $1.0_{0}$ & 8400 & $1.3_{0}$ \\
\hline
\end{tabular}

a Polymerization condition: BOL, $6.4 \mathrm{~g}$; K-Pyrdn, $1 \mathrm{~mol} \%$ to $\mathrm{BOL} ; \mathrm{Me}_{2} \mathrm{SO}, 20 \mathrm{~g}$; temperature, $25^{\circ} \mathrm{C}$; time, $30 \mathrm{~min}$.

b Reaction conditions: temperature, $25^{\circ} \mathrm{C}$; time, 5-6h.

c Degree of polymerization of the polymer determined by ${ }^{1} \mathrm{H}$ NMR spectroscopy.

d Number of terminal vinylbenzyl groups per polymer chain.

e Determined by ${ }^{1} \mathrm{H}$ NMR spectroscopy.

${ }^{f}$ Determined by UV spectroscopy using $N$-( $p$-vinylbenzyl)acetamide as a model compound $\left(\varepsilon_{\max }, 720\right.$ at $294 \mathrm{~nm}$ in chloroform).

g Determined by GPC.

Both contents of the benzamide and $p$ vinylbenzyl groups in the polymer were determined from the relative peak intensities in the expanded ${ }^{1} \mathrm{H}$ NMR spectrum. The degree of polymerization of the polyamide $(n)$ and the number of the vinylbenzyl group per polymer chain $(x)$ can be estimated using the following equation (eq 2), provided the benzamide group is located at one end of each polymer chain:

$$
\begin{aligned}
& (C+D):(E+F):\left(G+H_{1}+H_{2}\right) \\
& =(3+4 x): 2 x:(n+x)
\end{aligned}
$$

where $C, D, E, F, G, H_{1}$, and $H_{2}$ are the relative intensities of peaks c, d, e, f, g, h $\mathrm{h}_{1}$, and $\mathrm{h}_{2}$ in Figure 1, respectively.

As shown in Table $\mathrm{I}$, the value of $n$ was found to be controlled by the amount of 
BzBOL used as an activator. The value of $x$, which was measured also by UV spectroscopy as well as by ${ }^{1} \mathrm{H}$ NMR spectroscopy, was always unity independent of the amount of the activator used. Taking into account that the other possible groups such as carboxyl and acyllactam groups were not detected by the NMR spectroscopy, the acyllactam-type growing end derived from $\mathrm{BzBOL}$ was found not to be destroyed by any side reaction during the polymerization but to react quantitatively with VBA. Consequently, the desired vinylbenzyl-type polyamide macromers of which molecular weights were 3900,5400 , and 8400 , were ascertained to be prepared in the present systems.

\section{Radical Homopolymerization of the Polyamide Macromer}

The resulting polyamide macromers of various molecular weights were homopolymerized using AIBN as a catalyst in DMF at $60^{\circ} \mathrm{C}$ leading to give high molecular weight polymers in high conversion, as shown in Table II and Figure 2.

In the GPC curve of the whole polymer actually recovered (Figure 2B), the left peak must be assignable to the resulting polymer but the assignment of the right plateau seems to be quite ambiguous. In general, the degree of polymerization of the radical homopolymers of macromers should be not so high because of the low concentration of double bond in macromer. ${ }^{14}$ Asami et al. ${ }^{15}$ reported that radical homopolymerization of polytetrahydrofuran macromer at an extremely low macromer concentration $\left(<1.6 \times 10^{-3} \mathrm{~mol} \mathrm{l}^{-1}\right)$ yielded the polymer having no double bond of which the degree of polymerization (DP) was about unity. In the present runs, there seems to be no possibility of the formation of such a polymer of $\mathrm{DP}=1$ having no double bond since the initial macromer concentration was controlled to be higher $\left(0.03-0.04 \mathrm{~mol} \mathrm{l}^{-1}\right)$. Therefore, most of the polymer of DP $=1$ included in the whole polymer may be no more than the
Table II. Radical homopolymerization of polyamide macromers ${ }^{\mathrm{a}}$

\begin{tabular}{|c|c|c|c|c|c|}
\hline \multicolumn{2}{|c|}{ Macromer } & \multirow{2}{*}{$\frac{\mathrm{DMF}}{\mathrm{g}}$} & \multirow{2}{*}{$\frac{\text { Conversion }^{\mathrm{b}}}{\%}$} & \multirow{2}{*}{$\mathrm{DP}^{\mathrm{c}, \mathrm{d}}$} & \multirow{2}{*}{$M_{n} \times 10^{-4^{c}}$} \\
\hline$M_{n}$ & $\mathrm{~g}$ & & & & \\
\hline 3900 & 0.50 & 3.2 & 73 & 5.5 & 2.1 \\
\hline 5400 & 0.50 & 2.3 & 72 & 6.8 & 3.7 \\
\hline 8400 & 0.50 & 1.5 & 71 & 8.8 & 7.4 \\
\hline
\end{tabular}

a AIBN, 22-24 mol $\%$ to macromer; temperature, $60^{\circ} \mathrm{C}$; time, 2 days.

b Determined by gel permeation chromatography.

c Degree of polymerization of the resulting polymer.

d Estimated from GPC.

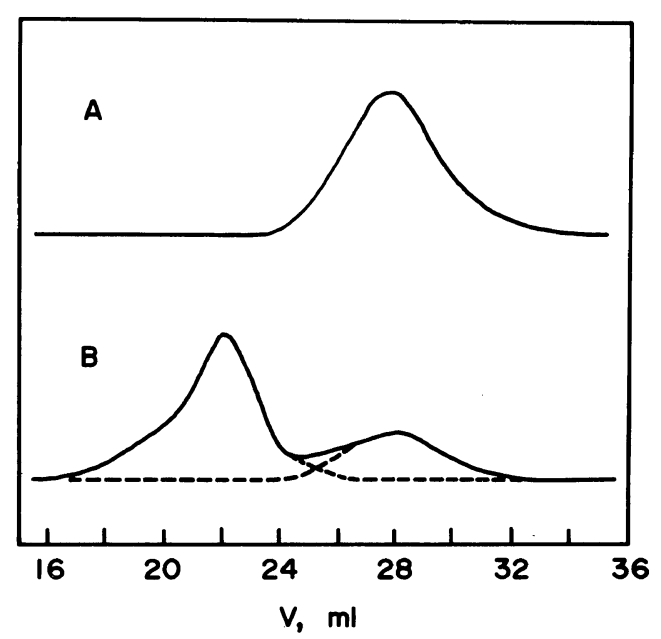

Figure 2. GPC curves of polymers (column, Shodex GPCA $803 \rightarrow 804,8 \phi \times 1000 \mathrm{~mm}$; solvent, chloroform). A, polyamide macromer $\left(M_{n}, 8400\right)$; $\mathrm{B}$, whole polymer recovered after the radical homopolymerization of the macromer.

unreacted macromer. The value of DP of the resulting homopolymer estimated by gel permeation chromatography was low as reported previously in other macromer systems. ${ }^{14-16} \mathrm{It}$ is also noteworthy that DP increased with an increase in the molecular weight of the macromer used.

Preparation of Graft Copolymer by Radical Copolymerization of the Polyamide Macromer with Styrene

The radical copolymerization of the mac- 
Table III. Radical copolymerization of polyamide macromers $\left(M_{1}\right)$ with styrene $\left(M_{2}\right)^{a}$

\begin{tabular}{|c|c|c|c|c|c|c|c|c|c|}
\hline \multicolumn{2}{|c|}{ Macromer } & \multirow{2}{*}{$\frac{\mathrm{M}_{2}}{\mathrm{~g}}$} & \multirow{2}{*}{$\frac{\begin{array}{c}\text { Polymer } \\
\text { yield }^{\mathrm{b}}\end{array}}{\mathrm{g}}$} & \multicolumn{2}{|c|}{$\frac{\text { Conversion }^{c}}{\%}$} & \multirow{2}{*}{$\frac{\begin{array}{c}\mathrm{M}_{1} \text { in } \\
\text { copolymer }\end{array}}{\mathrm{mol}_{\mathrm{o}}^{\mathrm{c}}}$} & \multirow{2}{*}{$\frac{1^{\mathrm{d}}}{r_{2}}$} & \multirow{2}{*}{$M_{n} \times 10^{-4^{\mathrm{e}}}$} & \multirow{2}{*}{$\begin{array}{l}\text { Number of } \\
\text { branches }^{f}\end{array}$} \\
\hline$M_{n}$ & in feed & & & $\mathbf{M}_{1}$ & $\mathbf{M}_{2}$ & & & & \\
\hline 3900 & 2.6 & 0.50 & 0.28 & 22 & 34 & 1.7 & 0.61 & 7.9 & 8.1 \\
\hline 3900 & 1.9 & 0.70 & 0.32 & 29 & 46 & 1.2 & 0.55 & 2.2 & 1.7 \\
\hline 3900 & 0.9 & 1.48 & 0.76 & 30 & 47 & 0.6 & 0.57 & 3.2 & 1.5 \\
\hline 5400 & 1.9 & 0.50 & 0.28 & 22 & 34 & 1.2 & 0.60 & 3.4 & 2.5 \\
\hline 5400 & 0.9 & 1.06 & 0.61 & 28 & 48 & 0.5 & 0.51 & 2.1 & 0.9 \\
\hline 8400 & 1.2 & 0.51 & 0.33 & 21 & 33 & 0.8 & 0.59 & 4.1 & 1.9 \\
\hline
\end{tabular}

${ }^{\mathrm{a}} \mathrm{M}_{1}, 0.50 \mathrm{~g}$; AIBN, $0.2 \mathrm{~mol} \%$ to monomer; DMF, $1.5 \mathrm{~g}$; temperature, $60^{\circ} \mathrm{C}$; time, 1 day.

b Insoluble in methanol-chloroform $(4: 1, \mathrm{v} / \mathrm{v})$ mixture.

c Determined by ${ }^{1} \mathrm{H}$ NMR spectroscopy.

d Reciprocal monomer reactivity ratio $\left(r_{2}=\ln \left(1-p_{2}\right) / \ln \left(1-p_{1}\right) ; p_{1}\right.$ and $p_{2}$ are conversions of $\mathbf{M}_{1}$ and $\left.\mathbf{M}_{2}\right)$.

e Determined by GPC (polystyrene standard).

f Calculated from the composition and molecular weight of the copolymer.

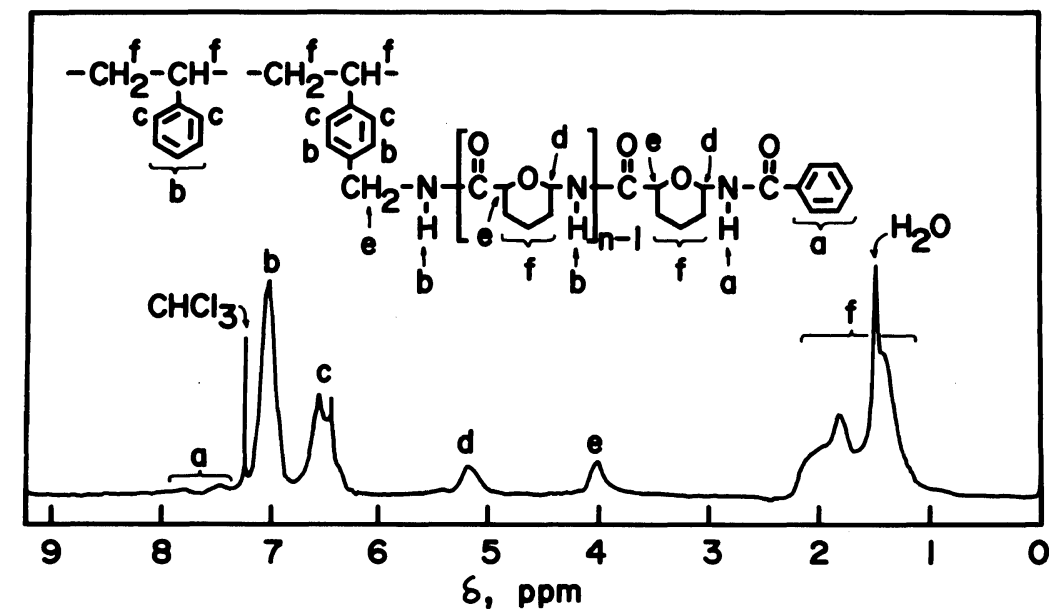

Figure 3. ${ }^{1} \mathrm{H}$ NMR spectrum of the graft copolymer obtained by radical copolymerization of the polyamide macromer $\left(M_{n}, 3900\right)$ with styrene $\left(M_{n}, 79000\right.$; mole fraction of BOL unit in the polymer, 0.33 ; $0.6 \%$ chloroform- $d$ solution; TMS; $50^{\circ} \mathrm{C} ; 200 \mathrm{MHz}$ ).

romer $\left(\mathbf{M}_{1}\right)$ with styrene $\left(\mathbf{M}_{2}\right)$ in different feed compositions proceeded homogeneously in DMF at $60^{\circ} \mathrm{C}$. The results are summarized in Table III. The resulting graft copolymer composed of a polystyrene stock and polyamide branches was easily separated from the unreacted macromer by the same fractional precipitation method as described in the previous communication. ${ }^{9}$ The number average molecular weight $\left(M_{n}\right)$ of the isolated graft copoly- mer was estimated by gel permeation chromatography using standard polystyrenes as references, but it may be underestimated because of the morphological characteristics of the graft copolymer.

In the ${ }^{1} \mathrm{H}$ NMR spectrum of the resulting graft copolymer were observed all peaks due to both polystyrene and polyamide segments as shown in Figure 3. The composition of the graft copolymer can be calculated from the 
relative peak intensities by using the following equation (eq 3) and listed in Table III.

$$
\begin{aligned}
& (B+C):(D+E) \\
& =[5(1-y)+(n+4) y]:[n y+(n+2) y]
\end{aligned}
$$

where $B, C, D$, and $E$ are the relative intensities of peaks b, c, d, and e in Figure 3, respectively. The symbols $y$ and $n$ represent the mole fractions of the macromer unit in the graft copolymer and the degree of polymerization of the polyamide, respectively. Some values of $y$ were determined also by the elemental analysis, which fairly agreed with those estimated by ${ }^{1} \mathrm{H}$ NMR spectroscopy with an error of maximum $10 \%$. They are always smaller than the corresponding feed compositions, which suggests that the apparent reactivity of the macromer may be lower than that of styrene.

The relative reactivity of the macromer with the styryl radical can be estimated by. the values of $1 / r_{2}$ calculated from the following equation (eq 4$)^{17}$ and shown in Table III.

$$
r_{2}=\ln \left(1-p_{2}\right) / \ln \left(1-p_{1}\right)
$$

where $p_{1}$ and $p_{2}$ are conversions of $\mathbf{M}_{1}$ and $\mathbf{M}_{2}$, respectively. The values are $0.5-0.6$ regardless of the molecular weight of the macromer in the present condition. The value of $0.56 \pm 0.13$ for the macromer of $M_{n} 3900$ was also obtained from the Mayo-Lewis' integral equation using the composition data in Table III. They are evidently lower than that of $p$-methylstyrene $\left(1 / r_{2}=1.2\right) .^{18}$

The reactivity difference in the copolymerization between a macromer and its model monomer of low molecular weight is one of the subjects involved in the study of macromers. It should be essentially negligible as confirmed by some investigators. ${ }^{19-25}$ On the other hand, Ito et al. ${ }^{26,27}$ indicated that the reactivities of acryl-type and vinylbenzyl-type polyoxyethylene macromers were lowered with increasing the chain lengths and explained by the "kinetic" excluded volume effect and the shield effect due to the aggregation of the polyoxyethylene chains on the reaction of the polymer radical with the macromer. Solvent effects on the radical copolymerization of macromers are also known to be significant. ${ }^{28-30}$ The essential reasons for the low copolymerizability of the polyamide macromer in the present system are ambiguous, but may be due to some kind of polymer effects derived from its polar and relatively hard polyamide branches.

\section{Characterization of Graft Copolymers}

The moisture sorption isotherm of the graft copolymer determined at $20^{\circ} \mathrm{C}$ on the coarsely ground sample, as shown in Figure 4, indicated that its hygroscopicity was significantly lower than that of the mixture of the hydrophilic homopolyamide and the nonpolar homopolystyrene, the composition of which was the same as that of the graft copolymer. The graft copolymer having relatively long polyamide branches had also the same tendency. This fact may be explained by the presumption that the polystyrene domains around the polyamide domains would prevent

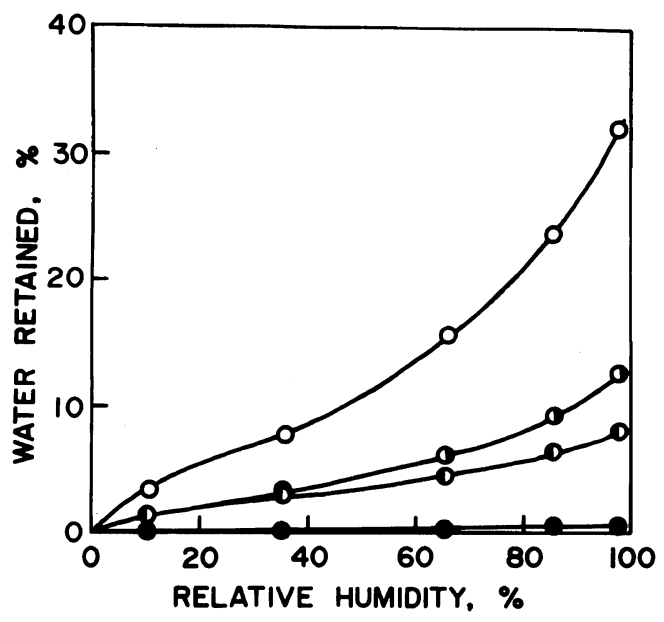

Figure 4. Moisture sorption isotherms of various polymers at $20^{\circ} \mathrm{C}$. $\bigcirc$, PolyBOL;, expected sorption of the mixture of polyBOL and polystyrene (weight fraction of polyBOL, 0.38); $D$, graft copolymer having a polystyrene stock and polyBOL branches (weight fraction of polyBOL branches $\left(M_{n}, 3600\right)$, $0.38)$;, polystyrene. 


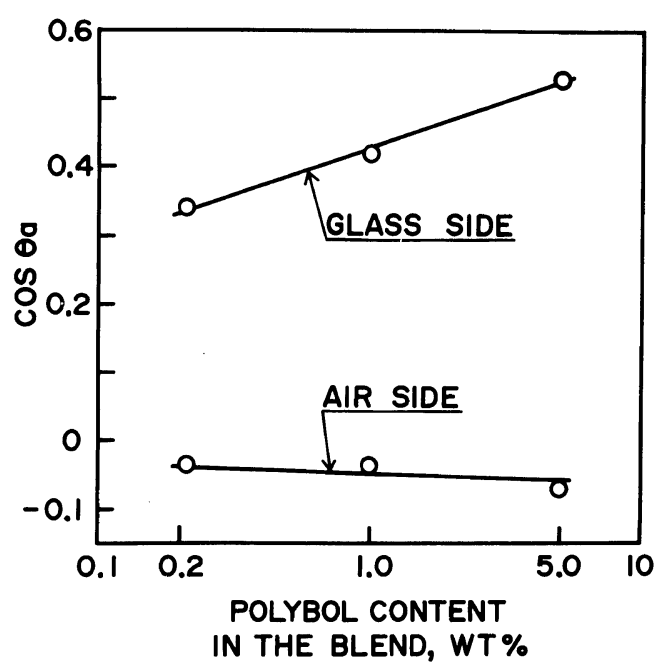

Figure 5. Contact angles of water droplets at $25^{\circ} \mathrm{C}$ on surfaces of the binary blend films composed of polystyrene and the graft copolymer $\left(M_{n}, 21000\right.$; weight fraction of polyBOL branches $\left.\left(M_{n}, 5100\right), 0.21\right)$.

the expansion of the latter due to water adsorption.

While the thermal transition point due to the fusion of the macromer $\left(M_{n} 3900\right)$ was $220-230^{\circ} \mathrm{C}$, no endothermic peak due to the fusion of the graft copolymer (weight fraction of polyBOL branches $\left.\left(M_{n}, 3600\right), 0.38\right)$ was observed at that temperature range by differential scanning calorimetry. The polyamide domains surrounded by polystyrene domains may be so small that the crystallization of the former domains should be disturbed by the surrounding latter domains.

The graft copolymer having the polyamide branches is soluble in various organic solvents such as chloroform, pyridine, DMF, and $\mathrm{Me}_{2} \mathrm{SO}$, in which the polyamide macromer is dissolved, although nylon 6 and nylon 4 are insoluble. Therefore the graft copolymer can be cast to brittle films from the chloroform solution.

Two kinds of the transparent films of binary blends, which were i) the graft copolymer and homopolystyrene and ii) the graft copolymer and polyBOL, were prepared by casting on a flat glass plate from the corresponding chlo-

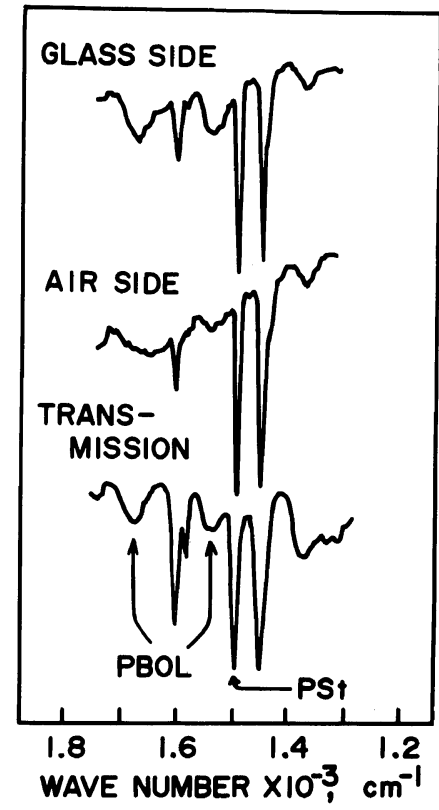

Figure 6. Attenuated total reflection-infrared spectra of the binary film composed of polystyrene and the graft copolymer $\left(M_{n}, 21000\right.$; weight fraction of polyBOL branches $\left.\left(M_{n}, 5100\right), 0.21\right)$. PolyBOL content in the blend, $1.0 \%$.

roform and chloroform-methanol $(9: 1)$ solutions, respectively. Figure 5 shows that the glass-side surface of the blend film comprising mainly polystyrene was made markedly hydrophilic by the addition of a small amount of the graft copolymer. Similar effects were observed in other blend systems. ${ }^{20,28}$

Thus, while the contact angle of a water droplet on the air-side surface of the blend film was almost the same as that on the hydrophobic homopolystyrene film $\left(91^{\circ}\right)$, the contact angle on the glass-side surface was effectively reduced from $91^{\circ}$ to $70^{\circ}$ by blending only $1 \%$ of the graft copolymer (the content of the polyamide segment in the blend film, $0.2 \%$ ). On blending $24 \%$ of the graft copolymer (the content of the polyamide segment, $5 \%$ ) the angle became $58^{\circ}$, which was essentially the same as the angle $59^{\circ}$ on the hydrophilic polyBOL film. This suggests that the hydrophilic polyamide segment in the blend film 


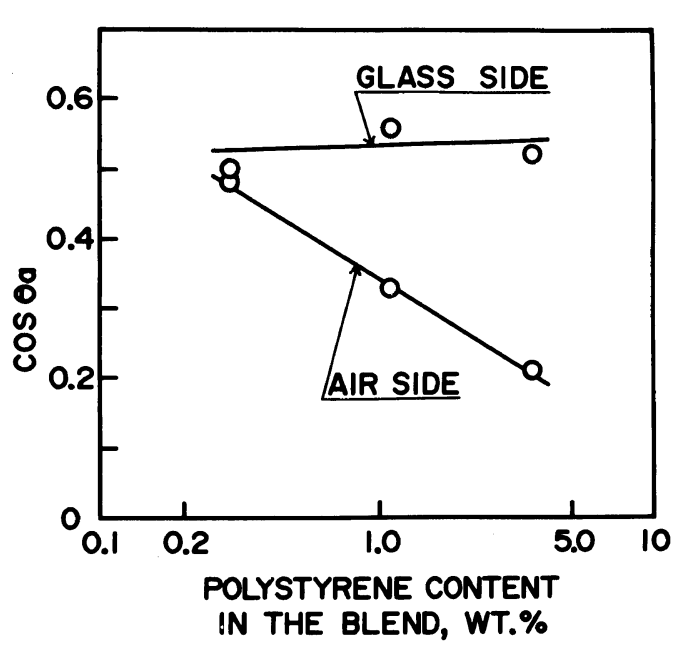

Figure 7. Contact angles of water droplets at $25^{\circ} \mathrm{C}$ on surfaces of the binary blend films composed of polyBOL and the graft copolymer $\left(M_{n}, 22000\right.$; weight fraction of polyBOL branches $\left.\left(M_{n}, 3600\right), 0.30\right)$.

was, at least partly, located and concentrated on the glass-side surface. This is also supported by the results of attenuated total reflection-infrared spectroscopy as seen in Figure 6.

In the case of the binary blend film containing mainly polyBOL, the glass-side surface is always hydrophilic, but the surface property of the air-side is changed with increasing the amount of the blended graft copolymer as shown in Figure 7 . Thus, the blending of $0.4 \%$ of the graft copolymer in the polyBOL film (the content of polystyrene segment in the blend, $0.3 \%$ ) was not so effective on the increase of the hydrophobicity of the air-side surface as indicated by the contact angle of $61^{\circ}$. Even if $5 \%$ of the graft copolymer was blended (the content of the polystyrene segment, $3.6 \%$ ) the angle increased only to $78^{c}$, which was much lower than that of the hydrophobic polystyrene $\left(91^{\circ}\right)$. The surface may not be completely covered with the polystyrene segment but with any micro-separated phases comprising polystyrene and polyBOL domains.

The vinylbenzyl-type polyamide macromer obtained in the present work can be copolymerized with other monomers to give various graft copolymers useful for polymeric composite materials.

Acknowledgement. The authors are grateful to Prof. Y. Yamashita and Dr. Y. Tsukahara of Nagoya University for kindly measuring the contact angles.

\section{REFERENCES}

1. H. Sumitomo and K. Hashimoto, Macromolecules, 10, 1327 (1977).

2. K. Hashimoto and H. Sumitomo, Macromolecules, 13, 786 (1980).

3. K. Hashimoto and H. Sumitomo, J. Polym. Sci., Polym. Chem. Ed., 21, 397 (1983).

4. H. Sumitomo, K. Hashimoto, and T. Ohyama, Polym. Bull., 1, 133 (1978).

5. H. Sumitomo, K. Hashimoto, and T. Ohyama, Polym. Bull., 1, 635 (1979).

6. H. Sumitomo and K. Hashimoto, "Contemporary Topics in Polymer Science," Vol. 4, Ed. by W. J. Bailey and T. Tsuruta, Plenum, New York, N. Y., 1984, p 779.

7. K. Hashimoto, H. Sumitomo, and H. Yamamori, Polym. J., 17, 679 (1985).

8. K. Hashimoto and H. Sumitomo, Polym. J., 15, 547 (1983).

9. K. Hashimoto, H. Sumitomo, and M. Kawasumi, Polym. Bull., 11, 121 (1984).

10. H. Sumitomo, K. Kobayashi, and K. Hashimoto, J. Macromol. Sci., Chem., A21, 1049 (1984).

11. H. Sekiguchi, Bull. Soc. Chim. Fr., 1827 (1960).

12. K. Kobayashi, H. Sumitomo, and Y. Ina, Polym. J., 15, 667 (1983).

13. A. J. Speziale, K. W. Ratts, and G. J. Marco, J. Org. Chem., 26, 4311 (1960).

14. R. Asami, M. Takaki, K. Kyuda, and E. Asakura, Polym. J., 15, 139 (1983).

15. R. Asami, M. Takaki, K. Kyuda, and N. Sukenaga, Polym. J., 15, 261 (1983).

16. J. Sierra-Vargas, E. Franta, and P. Rempp, Makromol. Chem., 182, 2603 (1981).

17. J. Kennedy and C. Y. Lo, Polym. Bull., 8, 63 (1982).

18. G. Natta, F. Danusso, and D. Sianesi, Makromol. Chem., 30, 238 (1959).

19. R. Milkovich, "Anionic Polymerization. Kinetics, Mechanism, and Synthesis," J. E. McGrath, Ed., ACS Symp. Ser., 166, 41 (1981) and references therein.

20. Y. Yamashita, Kobunshi, 31, 988 (1981).

21. K. Ito, N. Usami, and Y. Yamashita, Macro- 


\section{K. Hashimoto, H. Sumitomo, and M. Kawasumi}

molecules, 13, 216 (1980).

22. M. Takaki, R. Asami, H. Hanahata, N. Sukenaga, and K. Sogawa, Polym. Prepr. Jpn., 30, 860 (1981).

23. M. Maeda and S. Inoue, Makromol. Chem., Rapid Commun., 2, 537 (1981).

24. Y. Yamashita, K. Ito, H. Mizuno, and K. Okada, Polym. J., 14, 255 (1982).

25. T. Nishimura, M. Maeda, Y. Nitadori, and T. Tsuruta, Makromol. Chem., 183, 29 (1982).

26. K. Ito, K. Uchida, A. Hayashi, H. Tsuchida, T. Kitano, and E. Yamada, Preprints of 1st SPSJ
International Conference, Japan, 1984, p 77.

27. K. Ito, H. Tsuchida, A. Hayashi, T. Kitano, E. Yamada, and T. Matsumoto, Polym. Prepr. Jpn., 33, 1151 (1984).

28. K. Ito, Y. Masuda, T. Shintani, T. Kitano, and Y. Yamashita, Polym. J., 15, 443 (1983).

29. R. Asami, M. Takaki, K. Sogawa, and Y. Sakaguchi, Polym. Prepr. Jpn., 32, 1115 (1983).

30. G. O. Schulz and R. Milkovich, J. Polym. Sci., Polym. Chem. Ed., 22, 1633 (1984). 